\title{
SARS spreads new outlook on quarantine models
}

When governments in Asia and Canada imposed quarantine measures at the height of the severe acute respiratory syndrome (SARS) epidemic, most experts-particularly in the US-decried the tactics as outdated and ineffective (Nat. Med. 9, 487; 2003). With fresh data from the SARS outbreaks, however, many now say that isolating even a small percentage of those who have been exposed to the illness-but who are not yet ill-could help quell an outbreak.

"We're now reinventing a fourteenthcentury public health tool, and we have to do it in the context of the twenty-first century," says Martin Cetron, deputy director of the Division of Global Migration and Quarantine at the US Centers for Disease Control and Prevention (CDC). "Quarantine doesn't have to be airtight and under armed guard to be effective."

Based on a model of quarantine's impact on smallpox transmission, Cetron says that isolating just $50 \%$ of those exposed to the smallpox virus could significantly reduce disease transmission. This is in stark contrast to the $90-95 \%$ experts had previously cited.

The CDC reported in October that only a third of the 30,000 Beijing residents quarantined during the SARS outbreak faced significant risk of contracting the flu-like illness (MMWR 52, 1037-1040; 2003). According to the report, only residents who came into direct contact with a symptomatic patient were later infected; those who had cared for a SARS patient carried the highest risk.

At least in the case of SARS, therefore, experts say quarantines can be implemented more efficiently by focusing on a smaller swath of the population: those who have had direct contact with an ill patient.

"For quarantine to be an important tool, it's most useful if people are infectious prior to the onset of symptoms-and we don't have any evidence that that's the case with SARS," says Donald Low, microbiologist-in-chief at Mt. Sinai Hospital in Toronto. There is no data to support that someone infected with SARS can transmit the coronavirus during the incubation period-the time between exposure to the virus and the onset of symptoms. Patients become infectious as they enter the advanced and more symptomatic stages of the disease, giving scientists a window of several days in which to diagnose and iso-

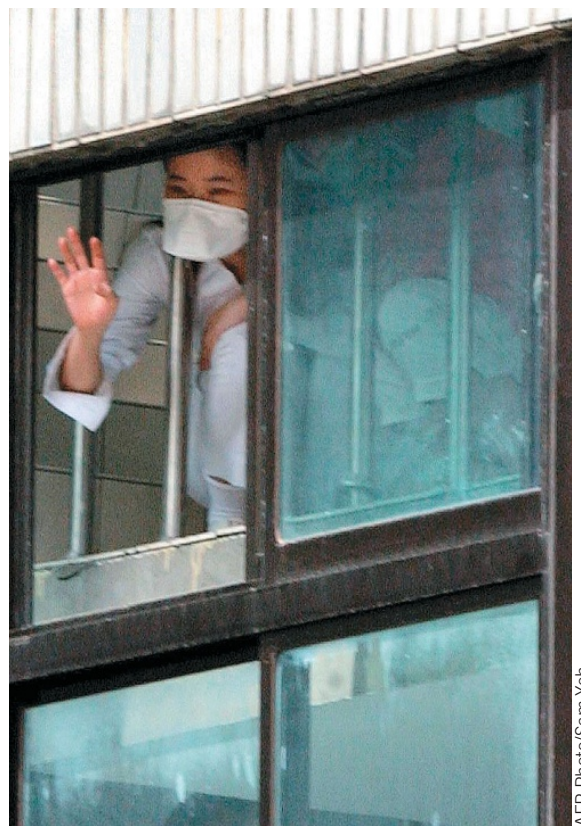

late infected people.

For that reason, Low says he now favors increased self-monitoring for early-onset symptoms, such as fever. Mathematical models can help provide a better understanding of when an infected person begins spreading SARS, Low adds.

Another factor is the virus' mode of transmission. The World Health Organization says SARS is transmitted almost exclusively through direct contact with infectious droplets. But not everyone is ready to accept that analysis.

Even if a tiny percentage of infections are transmitted through the air or some means other than droplet spread, says James Koopman, a University of Michigan epidemiologist, it could completely alter the virus' transmission pattern. Koopman is developing a model to address the relative significance of different transmission patterns.

"It could be that less than $5 \%$ of all transA grounded hospital employee waves from a window at the Taipei Municipal Hoping Hospital in April. Governments of many countries instituted mandatory quarantines during the SARS epidemic. missions are airborne," says Koopman. "But it may be that stopping that $5 \%$ is more important than stopping all the other 95\%."

Bruce Diamond, Washington, DC

\section{Report calls for overhaul of UK clinical trials}

The working group of the UK Academy of Medical Sciences in October issued a report calling for vast changes to the clinical research system, and criticized the UK National Health Service (NHS), one of the country's main sources of support for clinical trials.

The academy recommended the creation of an NHS-funded National Network for Clinical Research that would have the status of a special health authority. The network would support clinical trials and research on seven major disease areas, including cancer, neurodegenerative diseases, and cardiovascular disease and stroke.

Other recommendations include adhering to the NHS's original goal of spending $1.5 \%$ of its turnover on clinical research activities, and recruiting and retaining more clinical researchers.

The report's recommendations were published in the British Medical Journal. In the same issue, another paper studied clinical trials between 1980 and 2002 and concluded that support for randomized, controlled trials by the NHS - and to a lesser extent, the Medical Research Council and medical charities - had fallen in recent years. The paper's authors also said that the decline in the number of trials did not come with an increase in the size of trials or more multicenter collaborations.

The academy's report follows a one-year review of the system, says John Bell, Regius Professor of Medicine at Oxford University and a member of the working group. "We've gotten fantastic stuff coming from the biomedical arena, but unless it delivers something to the bedside, it will be under threat and we have to fix that problem," Bell says.

In a statement, the UK Department of Health (DOH), which oversees the NHS, said it would not comment until it saw another report commissioned by the $\mathrm{DOH}$ and the Department of Trade and Industry to make recommendations on the future of the UK's bioscience industry. That report raises similar issues, says Vicky Wyatt, a press officer at the NHS desk.

Aparna Surendran, New York 